\title{
Interneurons in the Honeybee Primary Auditory Center Responding to Waggle Dance-Like Vibration Pulses
}

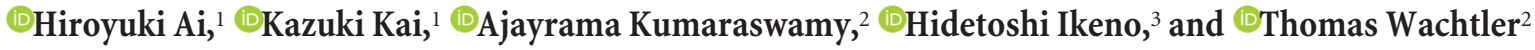 \\ ${ }^{1}$ Department of Earth System Science, Fukuoka University, Fukuoka 814-0180, Japan, ${ }^{2}$ Department of Biology II, Ludwig-Maximilians-Universität \\ München, 82152 Planegg-Martinsried, Germany, and 3School of Human Science and Environment, University of Hyogo, Himeji 670-0092, Japan
}

Female honeybees use the "waggle dance" to communicate the location of nectar sources to their hive mates. Distance information is encoded in the duration of the waggle phase (von Frisch, 1967). During the waggle phase, the dancer produces trains of vibration pulses, which are detected by the follower bees via Johnston's organ located on the antennae. To uncover the neural mechanisms underlying the encoding of distance information in the waggle dance follower, we investigated morphology, physiology, and immunohistochemistry of interneurons arborizing in the primary auditory center of the honeybee (Apis mellifera). We identified major interneuron types, named DL-Int-1, DL-Int-2, and bilateral DL-dSEG-LP, that responded with different spiking patterns to vibration pulses applied to the antennae. Experimental and computational analyses suggest that inhibitory connection plays a role in encoding and processing the duration of vibration pulse trains in the primary auditory center of the honeybee.

Key words: brain; dance language; honeybee; Johnston's organ; primary auditory center; vibration; waggle dance

\section{Significance Statement}

The waggle dance represents a form of symbolic communication used by honeybees to convey the location of food sources via species-specific sound. The brain mechanisms used to decipher this symbolic information are unknown. We examined interneurons in the honeybee primary auditory center and identified different neuron types with specific properties. The results of our computational analyses suggest that inhibitory connection plays a role in encoding waggle dance signals. Our results are critical for understanding how the honeybee deciphers information from the sound produced by the waggle dance and provide new insights regarding how common neural mechanisms are used by different species to achieve communication.

\section{Introduction}

Karl von Frisch demonstrated that honeybees use a type of movement called the "waggle dance" to direct their nest mates to a remote food source (von Frisch, 1967). The duration of the phase of the "waggle" movement changes linearly with the distance to the food source, suggesting that this distance information is en-

Received Jan. 5, 2017; revised Sept. 18, 2017; accepted Sept. 19, 2017.

Author contributions: H.A. and T.W. designed research; H.A., K.K., and A.K. performed research; A.K. contributed unpublished analytical tools; H.A., A.K., H.I., and T.W. analyzed data; H.A., A.K., H.I., and T.W. wrote the paper.

This research was supported by Grant-in-Aids for Scientific Research from the Ministry of Education, Science, Technology, Sports, and Culture of Japan (C, Grant No. 22570079); a grant for Challenging Exploratory Research (Grant 15K14569 to H.A.) from the Strategic International Cooperative Program, Japan Science and Technology Agency (JST); a grant from the German Federal Ministry of Education and Research (Grant 01GQ1116); and a grant from the Central Research Institute of Fukuoka University (Grant 151031). We thank Dr. Philipp Rautenberg for building a platform to share the database among our research group, Dr. Hidehiro Watanabe for technical advice regarding our experiments, and Drs. Azusa Kamikouchi and Yuki lshikawa for their critical comments concerning this research.

The authors declare no competing financial interests.

Correspondence should be addressed to Hiroyuki Ai, Division of Biology, Department of Earth System Science, Fukuoka University, 8-19-1 Nanakuma, Jonan-ku, Fukuoka 814-0180, Japan. E-mail: ai@fukuoka-u.ac.jp.

DOI:10.1523/JNEUROSCI.0044-17.2017

Copyright $\odot 2017$ the authors $\quad 0270-6474 / 17 / 3710624-12 \$ 15.00 / 0$ coded in a physical parameter of the movement that changes with the duration (von Frisch, 1967). Although it is possible that substrate-borne vibration elicited by the waggle movement conveys spatial information regarding the food source (Michelsen, 2003), air-borne vibration is thought to be the most probable sensory cue delivered during waggle dance communication (Judd, 1995) where wing beats produce local air-jet flows. During the waggle phase of the dance, the wingbeats of the dancer produce a train of vibration pulses that pass from the tail end of the dancer to a follower bee, which follows behind the dancer. For a fixed target location, the duration of the waggle phase is constant, as are pulse rate $(29 \mathrm{~Hz})$ and waggle frequency $(14.5 \mathrm{~Hz})$, independent of the quality of the food source (Hrncir et al., 2011). However, the precise feature of the train of vibration pulses elicited during the waggle dance that encodes distance information is unclear. Two plausible parameters are (1) the duration of the train of vibration pulses and (2) the number of vibration pulses per waggle phase. These parameters are linearly related because the rate of the pulsed vibration is nearly constant.

Waggle dance followers detect air-borne vibrations via Johnston's organ (JO), which is located at the second segment (pedi- 
cel) of the antenna (Towne and Kirchner, 1989; Kirchner et al., 1991; Dreller and Kirchner, 1993). The structural characteristics of the antenna and the response of JO neurons suggest that mature honeybee antennae and JO neurons are tuned to $250-300 \mathrm{~Hz}$, which matches the frequency of sound generated during the waggle dance (Tsujiuchi et al., 2007). JO afferent fibers are spatially segregated in the medial posterior protocerebral lobe (mPPL) and the dorsal lobe (DL)-dorsal subesophageal ganglion (dSEG; Ai et al., 2007). The dSEG also receives sensory afferents from neck hairs that are thought to be gravity sensors (Brockmann and Robinson, 2007). This suggests that the dSEG integrates vector information about the waggle dance (i.e., distance information coded in air vibrations and direction information coded in the orientation of the dancer relative to gravity; Ai and Hagio, 2013).

Our goal is to understand how distance and direction information is encoded in the honeybee brain. Accordingly, we are interested in characterizing the distribution and location of neurons that exhibit appropriate response properties for processing distance or direction information. Recently, we identified two interneuron (Int) types, DL-Int-1 and DL-Int-2, that receive JO input and are located in the primary auditory center (PAC) of the honeybee. We demonstrated that these interneurons are responsive to antennal stimulation (Ai et al., 2009; Ai, 2010; Ai and Itoh, 2012). In the present report, we describe the above-mentioned interneurons as well as a newly identified neuron type, the bilateral DL-dSEG-lateral protocerebrum (LP). We investigated the morphology, GABA immunoreactivity, and physiology of these three cell types in the PAC, with a particular focus on their responses to trains of vibration pulses with temporal properties similar to those elicited during the waggle dance. This work represents a first step toward understanding the role of these neurons in the encoding of distance information in the honeybee brain.

\section{Materials and Methods}

Preparation. Honeybees (Apis mellifera L., NCBITaxon:7460) were reared in hives placed on the Fukuoka University campus. The data reported here were collected between the months of April and September from 2013 to 2015. Female worker bees that were returning to the hive after foraging and had pollen on their hindlegs were caught at the hive entrance and used in this study.

Intracellular recording and staining. To investigate the processing of the sound produced during the waggle dance, we collected auditory interneurons in the PAC, including the previously described interneuron types DL-Int-1 and DL-Int-2 (Ai et al., 2009). The procedure for each subject was as follows. An individual bee was immobilized by placing it in a cold environment and then mounting it in an acrylic chamber. The bee was then given a $1 \mathrm{~m}$ sucrose solution as food and kept overnight in a dark room with high humidity and a temperature of $20^{\circ} \mathrm{C}$. The next day, the head of the bee was fixed in place with wax and the frontal surface of the brain was exposed by cutting away a small rectangular window between the compound eyes. The glands and tracheal sheaths on top of the brain were removed, and the mouthparts, including the mandibles, were cut off to expose and enable the removal of the esophagus. Small droplets of bee physiological saline (in mM: $137 \mathrm{NaCl}, 3 \mathrm{KCl}, 1 \mathrm{CaCl}_{2}, 4 \mathrm{Na}_{2} \mathrm{HPO}_{4}$, $2 \mathrm{KH}_{2} \mathrm{PO}_{4}, 100$ sucrose, $\mathrm{pH} 6.7$ ) were applied to wash away any residue in the esophagus and to enhance electrical contact with a platinum ground electrode placed in the head capsule next to the brain.

Borosilicate glass electrodes were pulled using a laser puller (P-2000, Sutter Instruments), and filled at the tip with 3\% Lucifer Yellow $\mathrm{CH}$ dilithium salt (catalog \#L0259, Sigma-Aldrich; PubChem substance accession identifier: 24896250 ) dissolved in $100 \mathrm{~mm} \mathrm{KCl}$, yielding DC resistances in the range of $150-300 \mathrm{M} \Omega$. We also used a dextrantetramethylrhodamine solution (3000 molecular weight, anionic, lysine fixable; catalog \#D3308, Thermo Fisher Scientific) and Alexa Fluor 647 hydrazide (catalog \#A20502, Thermo Fisher Scientific) for this injection. After removing a small section of the neural sheath and neurilemma, we inserted electrodes into the DL-dSEG and the mPPL and began recording electrical activity from individual neurons. Electrical signals were amplified with an amplifier (MEZ 8301, Nihon Kohden) and were displayed on an oscilloscope. Data were recorded and analyzed using the data acquisition and analysis software Spike2 (Cambridge Electronic Design; RRID: SCR_000903). After recording electrical activity, we applied a hyperpolarizing current (2-5 nA for 2-10 $\mathrm{min}$ ) to fill the neurons with Lucifer yellow. Thereafter, the brains were dissected out, fixed in $4 \%$ paraformaldehyde for $4 \mathrm{~h}$ at room temperature, then rinsed in phosphate buffer solution, dehydrated, and cleared in methyl salicylate for subsequent observation.

To identify vibration-sensitive neurons, we applied a continuous vibration to the right antenna and recorded the responses intracellularly. When a neuron was responsive to the vibration, we applied a continuous vibration at different frequencies. These frequencies included $265 \mathrm{~Hz}$, which is the fundamental frequency of the waggle dance sound. We also applied single vibration pulses and trains of vibration pulses with varying pulse durations and intervals (see below). When the recording became unstable (e.g., fluctuating membrane potential or spike amplitude), we stopped applying the vibration stimuli and injected fluorescence dye into the neuron for morphological analysis.

Sensory stimulation. First, both antennal scapes of each honeybee were fixed to the acrylic chamber with wax. For vibratory stimulation of the JO, the right antenna was inserted into a glass capillary (length, $10 \mathrm{~mm}$; inner diameter of the tip, $200 \mu \mathrm{m}$ ) up to the second segment of the flagellum and then fixed to the tip of the capillary with wax. The opposite tip of the capillary was connected to a piezo-actuator (Miniature Piezo Flexure NanoPositioners, catalog \#P780.20, PI Japan). The piezo-actuator was programmed to move with a specific temporal pattern, which was controlled using the Spike2 software. This movement created a vibratory stimulation. We used vibrations with frequencies ranging from 100 to $400 \mathrm{~Hz}$ and amplitudes (peak to peak) ranging from 0 to $50 \mu \mathrm{m}$ and 10 or 20 trains of vibration pulses with the interval ranging from 20 to $100 \mathrm{~ms}$ and with the duration ranging from 4 to $50 \mathrm{~ms}$. The recorded neural activities and signals sent to the actuator were simultaneously recorded and stored on a PC.

GABA immunocytochemistry. After identifying the interneurons that had been filled with Lucifer yellow dye, the preparations including our marked target neurons were selected for GABA immunocytochemistry. The preparations were rehydrated through a series of ethanol steps and $0.1 \mathrm{~m}$ PBS containing $0.5 \%$ Triton X-100 (PBST), pH 7.4. The tissue was then sliced into $100-\mu \mathrm{m}$-thick vibratome sections. Next, the sections were incubated in 5\% normal goat serum (NGS; catalog \#G9023, SigmaAldrich) in PBST (PBST-NGS) for $1 \mathrm{~h}$ at room temperature to block nonspecific staining and then incubated in primary antiserum, rabbit anti-GABA (1:1000 in PBST-NGS; catalog \#A2052, Sigma-Aldrich; RRID:AB_477652), and mouse anti-SYNORF1 (1:1000 in PBST-NGS; DSHB 3C11; RRID:AB_528479) for $3 \mathrm{~d}$ at $4^{\circ} \mathrm{C}$ in a shaking incubator. The anti-GABA was produced using GABA-bovine serum albumin (BSA; catalog \#A2153-10G; Sigma-Aldrich) as the immunogen. The antibody was isolated from the antiserum using immunospecific methods of purification. Antigen-specific affinity isolation removes essentially all rabbit serum proteins, including Igs that do not specifically bind to GABA. Rabbit anti-GABA exhibited positive binding with GABA and GABA-keyhole limpet hemocyanin in a dot blot assay, and negative binding with BSA. We used $1 \mathrm{~mm}$ GABA to remove all background staining after preadsorption of the primary antibody. The sections were then washed in PBST and incubated in an Alexa Fluor 647-conjugated goat anti-rabbit IgG antibody (1:1000 in PBST-NGS; catalog \#A11034, Thermo Fisher Scientific; RRID:AB_2576217) and an Alexa Fluor 555 goat antimouse IgG antibody (1:1000 in PBST-NGS; catalog \#A21424, Thermo Fisher Scientific; RRID:AB_141780) overnight at $4^{\circ} \mathrm{C}$. As controls, some preparations were processed without the anti-GABA antibody. Finally, after washing, the sections were mounted on slide glasses using VECTASHIELD (catalog \#H-1000, Vector Laboratories; RRID:AB_2336789).

Confocal microscopy. The cleared specimens containing intracellularly stained neurons were viewed from the posterior side of the brain under a confocal laser-scanning microscope (LSM 510, Carl Zeiss) with a Zeiss Plan-Apochromat $25 \times$ /numerical aperture (NA) 0.8 oil lens objective 
Table 1. List of PAC interneurons

\begin{tabular}{|c|c|c|c|c|c|c|}
\hline Category & Group name & Neuron name & Arborized neuropils & Response pattern to continuous vibration & $N_{\text {total }}$ & $\overline{N_{\text {ana }}}$ \\
\hline \multirow[t]{7}{*}{ Local interneurons } & DL-Int-1 & DL-Int-1 & DL, dSEG, mPPL & On-off phasic E and tonic I & 36 & 14 \\
\hline & \multirow[t]{4}{*}{ DL-dSEG-mPPL } & DL-dSEG-mPPL1 & \multirow[t]{4}{*}{ DL, dSEG, mPPL } & Tonicl & 17 & 6 \\
\hline & & DL-dSEG-mPPL2 & & Tonic E & 3 & 2 \\
\hline & & DL-dSEG-mPPL4 & & On-phasic I & 1 & 0 \\
\hline & & DL-dSEG-mPPL5 & & No response & 3 & 0 \\
\hline & DL-dSEG & DL-dSEG & DL, dSEG & Tonic l & 5 & 2 \\
\hline & DL-local & DL-local2 & DL & Tonicl & 1 & 1 \\
\hline \multirow[t]{5}{*}{ Output neurons } & DL-Int-2 & DL-Int-2 & $D L, d S E G, L P, C P P L$ & On phasic $\mathrm{E}$ or tonic $\mathrm{E}$ & 14 & 5 \\
\hline & DL-LP & DL-LP & $\mathrm{DL}, \mathrm{LP}$ & Tonic l & 6 & 1 \\
\hline & \multirow[t]{3}{*}{ DL-dSEG-LP } & DL-dSEG-LP1 & \multirow[t]{3}{*}{ DL, dSEG, LP } & Tonic I & 3 & 2 \\
\hline & & DL-dSEG-LP2 & & On-phasic E & 2 & 0 \\
\hline & & DL-dSEG-LP3 & & No response & 1 & 0 \\
\hline \multirow{3}{*}{ Bilateral neurons } & Bilateral DL-dSEG-mPPL & Bilateral DL-dSEG-mPPL & $\mathrm{DL}, \mathrm{dSEG}, \mathrm{mPPL}$ & Phasic tonic E & 1 & 1 \\
\hline & Bilateral DL-dSEG-PPL & Bilateral DL-dSEG-PPL & DL, dSEG, PPL & Tonic E & 1 & 1 \\
\hline & Bilateral mPPL-LP & Bilateral mPPL-LP & $\mathrm{mPPL}, \mathrm{LP}$ & Tonic I & 2 & 1 \\
\hline
\end{tabular}

$N_{\text {total }}$ Number of preparations of PAC neurons responding to vibration stimuli; $N_{\text {anar }}$ Number of preparations with sufficient response data used for analysis; E, excitation; I, inhibition; CPPL, central posterior protocerebral lobe.

(working distance, $0.57 \mathrm{~mm}$ ) for low-magnification images or with a Zeiss Plan-Apochromat 40×/NA 1.4 oil lens objective (working distance, $0.13 \mathrm{~mm}$ ) for high-magnification images. Alexa Fluor 555 and Alexa Fluor 647 were excited by the 543 and $633 \mathrm{~nm}$ spectral lines of an $\mathrm{HeNe}$ laser, respectively, while Lucifer yellow was excited by the $488 \mathrm{~nm}$ spectral line of an argon laser. Optical sections were made at $3 \mu \mathrm{m}$ (in low-magnification images) or $1.5 \mu \mathrm{m}$ (in high-magnification images) throughout the entire depth of each specimen. The image resolution was $1024 \times 1024$ pixels.

Nomenclature. We use the terms proposed by Mobbs (1982) and Milde (1988) to describe the neuropilar regions in the SEG of the honeybee brain. The orientation of neuronal structures is given according to the body axis.

Experimental design and statistical analysis. To evaluate the significant responses induced by vibration of the antenna, we calculated timeresolved spike frequency rates before $(0.5 \mathrm{~s}$ before the onset of each train), during, and after the stimulation, and statistically analyzed the differences between the responses (Wilcoxon signed rank test) using the software R version 3.4.0 (RRID:SCR_001905). To characterize the responses to different temporal patterns of pulse stimulation, we calculated the spike numbers, spike frequency, latency of the first spike, EPSPs, and IPSPs after the pulse stimulus for different pulse durations and intervals, and statistically analyzed the differences (Wilcoxon signed rank test). The criterion for all statistical tests was $p<0.01$.

Modeling and simulation. Membrane potentials of DL-Int-1 and DLInt-2 neurons were modeled using the Adaptive Exponential Integrateand-Fire (AdExp) model (Brette and Gerstner, 2005; Fig. 8-1 available at https://doi.org/10.1523/JNEUROSCI.0044-17.2017.f8-1). Membrane potential calculations included synaptic input currents, which were calculated from synaptic conductances. Synaptic conductances were simulated using difference-of-exponentials functions (Fig. 8-1C available at https://doi.org/10.1523/JNEUROSCI.0044-17.2017.f8-1), based on the Exp2Syn model of the simulator NEURON (Carnevale and Hines, 2006; RRID:SCR_005393). Neuron and synapse parameters were adjusted to qualitatively reproduce the response properties of DL-Int-1 and DL-Int-2 neurons as determined in our electrophysiological experiments (Fig. 8-1 B,D available at https://doi.org/10.1523/JNEUROSCI. 0044-17.2017.f8-1). The network of DL-Int-1 and DL-Int-2 neurons was implemented using the simulator Brian version 2.0.1 (Stimberg et al., 2014; RRID:SCR_002998) in Python. The code is available at https://web. gin.g-node.org/ajkumaraswamy/HB-PAC_disinhibitory_network (doi: 10.12751/g-node.1090f8). An integration step size of $0.1 \mathrm{~ms}$ was used, and all simulation runs had a "settling time" of $600 \mathrm{~ms}$, after which stimuli were applied. Network inputs were modeled as spikes from JO sensory neurons spiking at the positive peak of the sinusoidal antennal vibration stimulus. Like the stimuli in our experiments, model stimuli were continuous sinusoidal vibrations with a frequency of $265 \mathrm{~Hz}$ and trains of sinusoidal pulses of $265 \mathrm{~Hz}$ vibrations with $16 \mathrm{~ms}$ pulse durations and $33 \mathrm{~ms}$ interpulse intervals (IPIs), values close to those observed in the honeybee waggle dance. In addition, pulse trains with $100 \mathrm{~ms}$ IPIs were used in the simulations to investigate the model results for longer pulse intervals, as used in the experiments.

\section{Results \\ Categories of vibration pulse-sensitive interneurons arborizing in the PAC}

We identified 119 vibration-sensitive interneurons arborizing in the PAC, DL, dSEG, and/or mPPL (Table 1). Of those, DL-Int-1 and DL-Int-2 neurons have been described previously (Ai et al., 2009), but the results reported here were obtained from neurons collected exclusively for the present study. We categorized the interneurons based on their arborization and on their response patterns to continuous vibration. Based on their general arborizing features, they were classified into the following three categories: PAC local interneurons, PAC output neurons, and PAC bilateral neurons.

\section{PAC local interneurons}

PAC interneurons had arborizations mainly inside the PAC (i.e., in the DL, dSEG, and/or mPPL). Based on arborization patterns, we identified five groups of neurons. These were further divided into subgroups based on response patterns (Table 1). Neurons in the largest group, DL-Int-1, showed arborizations in the DL, $\mathrm{dSEG}$, and mPPL, and showed on-off phasic excitation and tonic inhibition to continuous vibration stimuli applied to the ipsilateral antenna. While DL-dSEG-mPPL neurons arborized in the same neuropils as DL-Int-1 neurons, individual DL-dSEG-mPPL neurons showed different response patterns to vibration stimuli, such as tonic inhibition, tonic excitation, on-phasic excitation, on-off phasic inhibition, or no response (Table 1). We found several other minor neuron groups in this category that arborized 

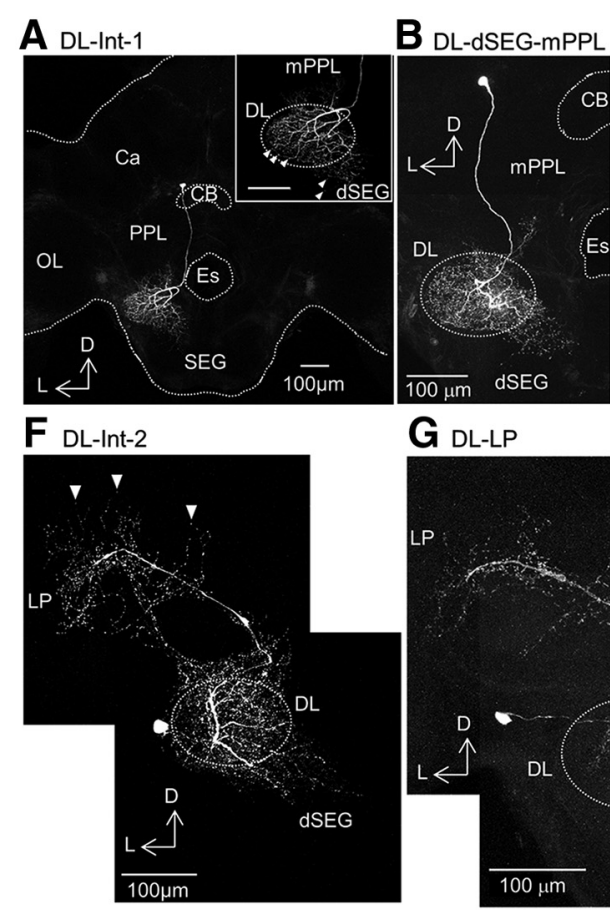

J Bilateral DL-dSEG-LP

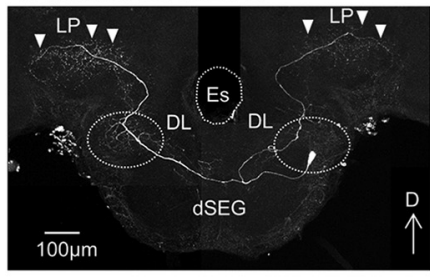

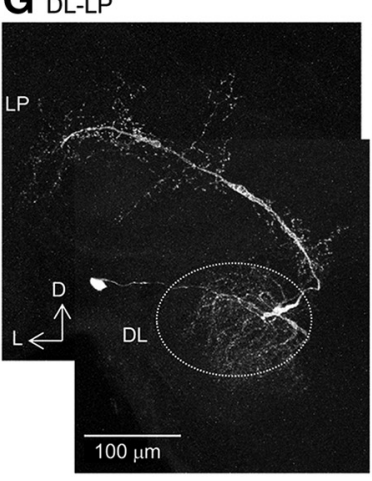

K Bilateral DL-dSEG-mPPL

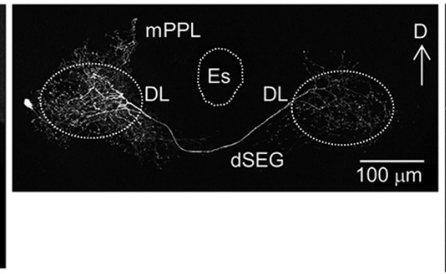

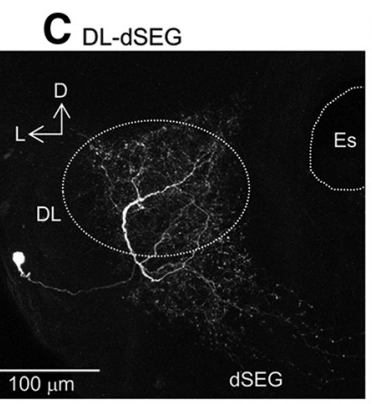

H DL-dSEG-LP

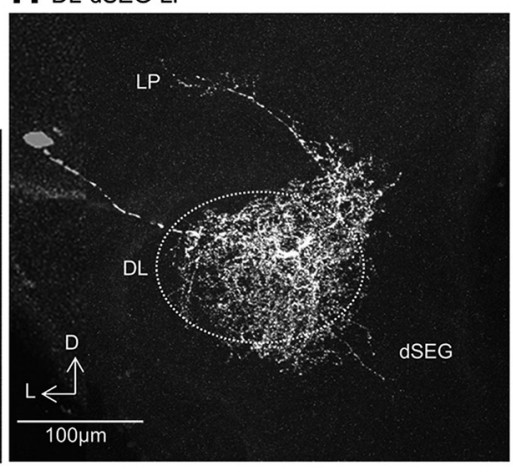

L Bilateral DL-dSEG-PPL

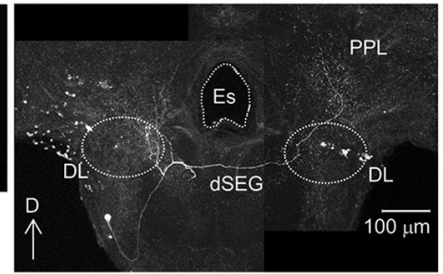

$\mathbf{D}_{\text {DL-mPPL }}$

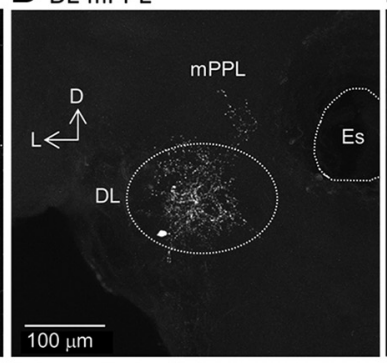

E DL local

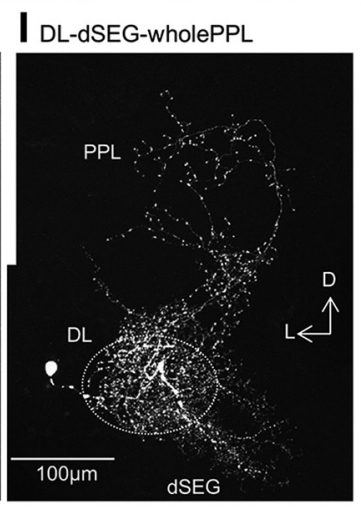

M Bilateral mPPL-LP

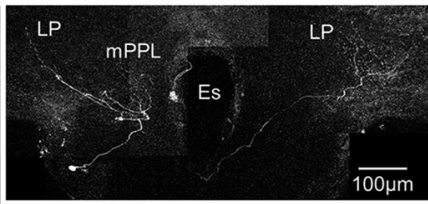

Figure 1. Morphology of PAC interneurons. Stained examples of the different neuron types investigated in this study (Table 1). $\boldsymbol{A}-\boldsymbol{E}$, Local interneurons arborized in the PAC neuropils DL, dSEG, and $\mathrm{mPPL}$. $\boldsymbol{A}, \mathrm{DL}-\mathrm{Int}-1$. This neuron type has dense arborizations in the DL and dSEG with fine spines and boutons (arrowhead), while in the medial PPL it has fine spines. The inset shows a magnification of the DL region. $\boldsymbol{B}-\boldsymbol{E}$, Four neuron types were named DL-dSEG-mPPL, DL-dSEG, DL-mPPL, and DL local according to the neuropils of arborization. $\boldsymbol{F}-\boldsymbol{I}$, Output neurons arborized in the PAC. Output neurons have dendritic arborizations in the PAC neuropiles, DL, and/or dSEG, and axon terminals in the LP and/or PPL. F, DL-Int-2 has a soma in the lateral cell cluster of the DL and arborizes with fine spines in the DL and dSEG, and with boutons in the LP (arrowheads). G-I, Three neuron types named DL-LP, DL-dSEG-LP, and DL-dSEG-wholePPL according to the neuropils of arborization. J-M, Bilateral neurons arborized in the PAC. Bilateral neurons have dendritic arborization in the bilateral PAC, DL, dSEG, or $\mathrm{mPPL}$. J, Bilateral DL-dSEG-LP neurons have symmetrical arborization in both the DL and LP. This neuron type has a spine branch in the DL-dSEG and terminals with boutons in the LP (arrowheads). $K-M$, Three neuron types named bilateral DL-dSEG-mPPL, bilateral DL-dSEG-PPL, and bilateral mPPL-LP according to the neuropils of arborization.

in different neuropils in the PAC (DL-dSEG, DL-mPPL, and DL local).

\section{PAC output neurons}

PAC output neurons had arborizations in the primary auditory center and sent their axons into the other neuropils of the PAC. Four groups were identified. Neurons in the main group, DL-Int-2, had arborizations in the DL and dSEG and sent their axons to the secondary auditory center, the LP. They showed excitatory responses to continuous vibration stimuli applied to the ipsilateral antenna. Moreover, we found several other minor neuron groups in this category (DL-LP, DL-dSEG-LP, and DL-whole PPL; Table 1).

\section{PAC bilateral neurons}

PAC bilateral neurons had arborizations in the PACs of both hemispheres and also sent their axons to the other neuropils in the PAC. Four groups were identified. Neurons in the main group, termed the bilateral DL-dSEG-LP, were newly identified in this study. These neurons had arborizations in both the DL and dSEG and sent their axons to both LPs. They showed on-phasic excitation to continuous vibration stimuli applied to the ipsilateral antenna. We found several other minor neuron groups in this category (bilateral DL-dSEG-mPPL, bilateral DL-dSEG-PPL, and bilateral mPPL-LP; Table 1).

\section{Categories of vibration pulse-sensitive interneurons arborizing in the PAC}

In 48 of the 119 preparations, the recording was stable for sufficiently long to apply a number of stimulation pulses with different combinations of vibration frequency, pulse duration, and pulse intervals. The data from those 48 neurons were used for further analysis. Of those, 27 neurons were from one of the main groups, DL-Int-1, DL-Int-2, or bilateral DL-dSEG-LP. We focused on these neurons for our analysis. While we report findings for neurons from the other groups as well, in many cases, only one or two examples of these interneurons were recorded (Table 1).

\section{Morphologies of PAC interneurons}

The main PAC local interneurons, termed DL-Int-1 and DLdSEG-mPPL (Fig. 1 $A, B$ ), connected the following neuropils in the PAC: the DL, the dSEG, and the MPPL. DL-Int-1 neurons had two branches. One branch arborized with fine terminals in the mPPL. The other branch arborized with fine terminals as well as 

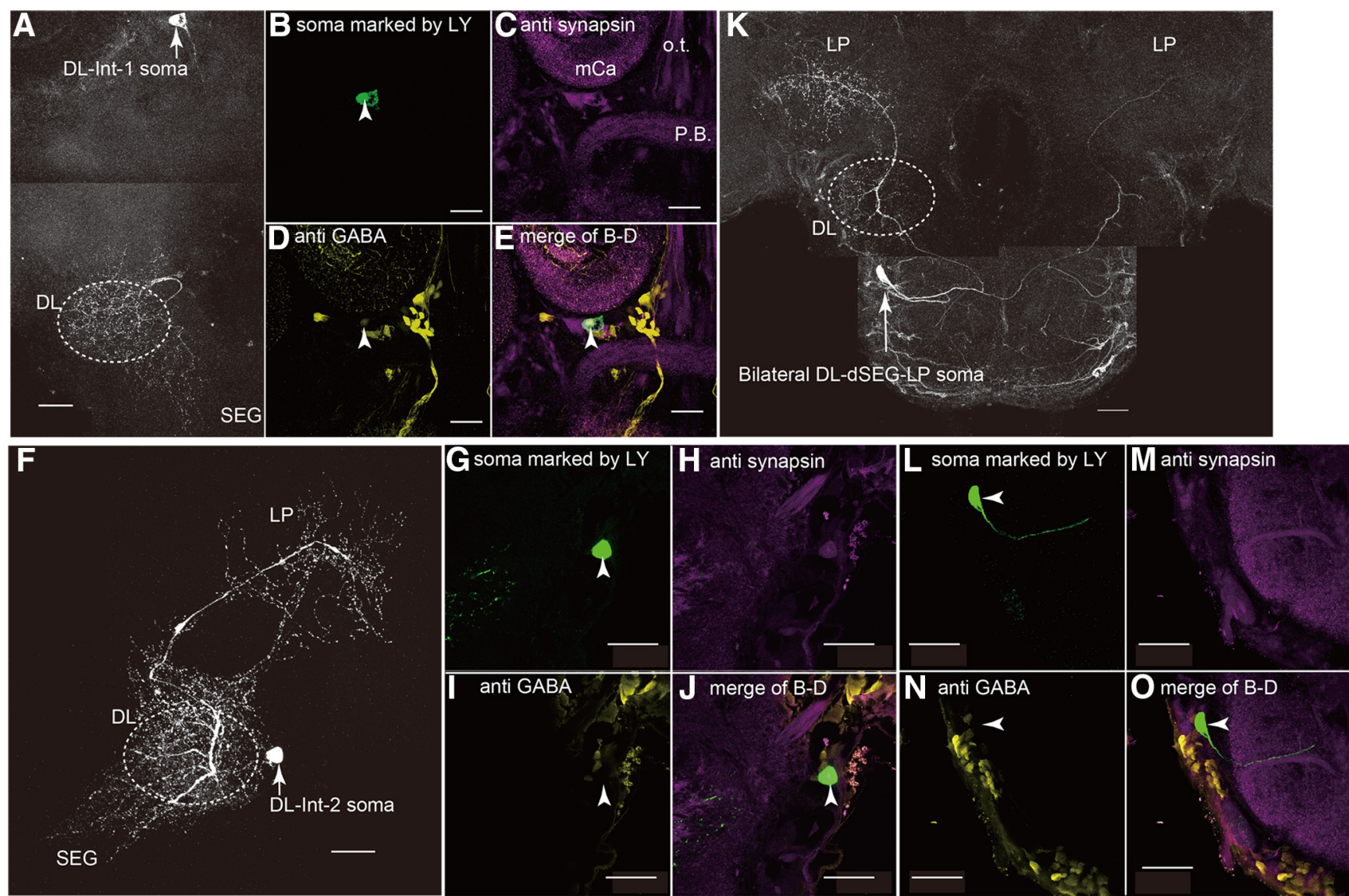

Figure 2. GABA immunoreactivity of PAC interneurons. Immunohistochemically stained examples of DL-Int-1, DL-Int-2, and bilateral DL-dSEG-LP neurons. $A$, Whole morphology of the DL-Int- 1 neuron (preparation HB130822-1). $\boldsymbol{B}-\boldsymbol{E}$, GABA immunoreactivity of DL-Int-1; the same optical section is visualized using different staining techniques. $\boldsymbol{B}$, Image of $L Y$-injected soma of DL-Int-1 neuron. $\boldsymbol{C}$, Anti-synapsin labeling. $\boldsymbol{D}$, Anti-GABA labeling. $\boldsymbol{E}$, Merged images of $\boldsymbol{B}-\boldsymbol{D}$. Arrows indicate the position of the DL-Int-1 soma. An anti-GABA-labeled spot coincides with the soma, suggesting that the DL-Int-1 is GABAergic. mCa, Medial calyx; 0.t., ocellar tract; P.B., protocerebral bridge. $F$, Whole morphology of DL-Int-2 (preparation 131217-2). G-J, GABA immunoreactivity of DL-Int-2; the same optical section is visualized using different staining techniques. G, DL-Int-2 soma. H, Anti-Synapsin labeling. I, Anti-GABA labeling.J, Merged images of G-I. The DL-Int-2 soma location (arrow in $\boldsymbol{G}$ and $\boldsymbol{I}$ ) does not overlap with GABA immunoreactivity, suggesting DL-Int-2 is not GABAergic. $\boldsymbol{K}$, Whole morphology of a bilateral DL-dSEG-LP neuron (preparation 130612-3). $\mathbf{L}-\mathbf{0}$, GABA immunoreactivity of bilateral DL-dSEG-LP; the same optical section is visualized using different staining techniques. $\boldsymbol{L}$, Bilateral DL-dSEG-LP soma. $\boldsymbol{M}$, Anti-Synapsin labeling. $\boldsymbol{N}$, Anti-GABA labeling. $\mathbf{0}$, Merged images of $\boldsymbol{L}-\boldsymbol{N}$. The bilateral DL-dSEG-LP soma does not overlap with GABA immunoreactivity, suggesting that the bilateral DL-dSEG-LP is not GABAergic.

boutons in the DL and dSEG (Fig. 1A, inset). DL-dSEG-mPPL neurons had a similar arborization pattern. The somata of DLInt-1 neurons were located dorsally from the central body, while the somata of DL-dSEG-mPPL neurons were located more laterally (Fig. $1 A, B$ ). The other local interneurons arborized in subsets of the PAC neuropils. DL-dSEG neurons had arborizations in the DL and dSEG but not in the mPPL. DL-mPPL neurons had arborizations in the DL and mPPL but not in the dSEG. DL-local neurons arborized inside the DL (Fig. $1 C-E$ ).

The PAC output neurons sent axons from the PAC to the other neuropils, mainly LP and PPL (Fig. $1 F-I$ ). All of the soma positions were located in the lateral DL. DL-Int-2 neurons had dense arborization in the DL-dSEG, with fine spines, and sparse and diverse arborization in the LP with boutons (Fig. 1F).

PAC bilateral neurons had dendritic arborizations in a PAC neuropil (either DL or mPPL) and sent processes to both hemispheres of the brain. Most of these neurons had arborizations in the DL, while two of them had arborizations in the mPPL, but not in the DL (Table 1). The neurons in this group arborized in the DL, dSEG, LP, and PPL. Most of these neurons were categorized as bilateral DL-dSEG-LP. Bilateral DL-dSEG-LP neurons had symmetrical arborizations in the DLs and LPs of both hemispheres, with fine spines in the DL and sparse and diverse ar- borization in the LP, as well as boutons (Fig. $1 J$ ). We also found several other minor groups with different arborization patterns in this category (bilateral DL-dSEG-mPPL, bilateral DL-dSEGPPL, and bilateral mPPL-LP; Fig. $1 K-M)$.

\section{GABA-immunohistochemistry of PAC interneurons}

We performed GABA immunohistochemical experiments with the PAC interneuron preparations (Fig. 2). The soma of each DL-Int-1 neuron was clearly visible in the dorsoposterior protocerebrum (Fig. 2A), and the locations of these neurons coincided with a GABA-immunoreactive spot, indicating that DL-Int-1 neurons are anti-GABA positive (Fig. $2 B-E$ ). In contrast, the soma locations of DL-Int-2 (Fig. $2 F-J$ ) and bilateral DL-dSEG-LP neurons (Fig. $2 \mathrm{~K}-\mathrm{O}$ ) did not overlap with a GABA-immunoreactive spot, indicating that DL-Int-2 and bilateral DL-dSEG-LP neurons are not anti-GABA positive.

\section{Responses of PAC interneurons to trains of vibration pulses}

Neurons of one of the major PAC local interneuron types, DL-Int-1, exhibited spontaneous activity, with rates ranging between 0 and $80 \mathrm{~Hz}$, depending on the preparation (Ai et al., 2009). When a train of vibration pulses was applied to the antenna, DL-Int-1 neurons showed tonic inhibition (Fig. 3A): The spike rate was suppressed 
A

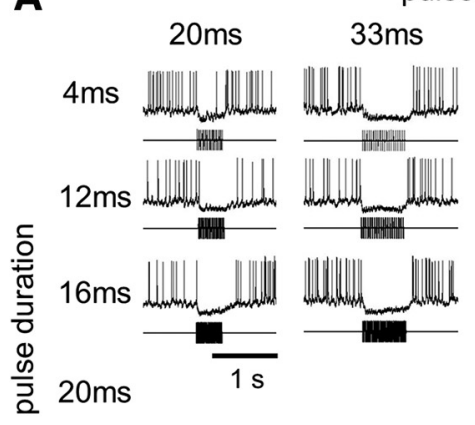

pulse interval

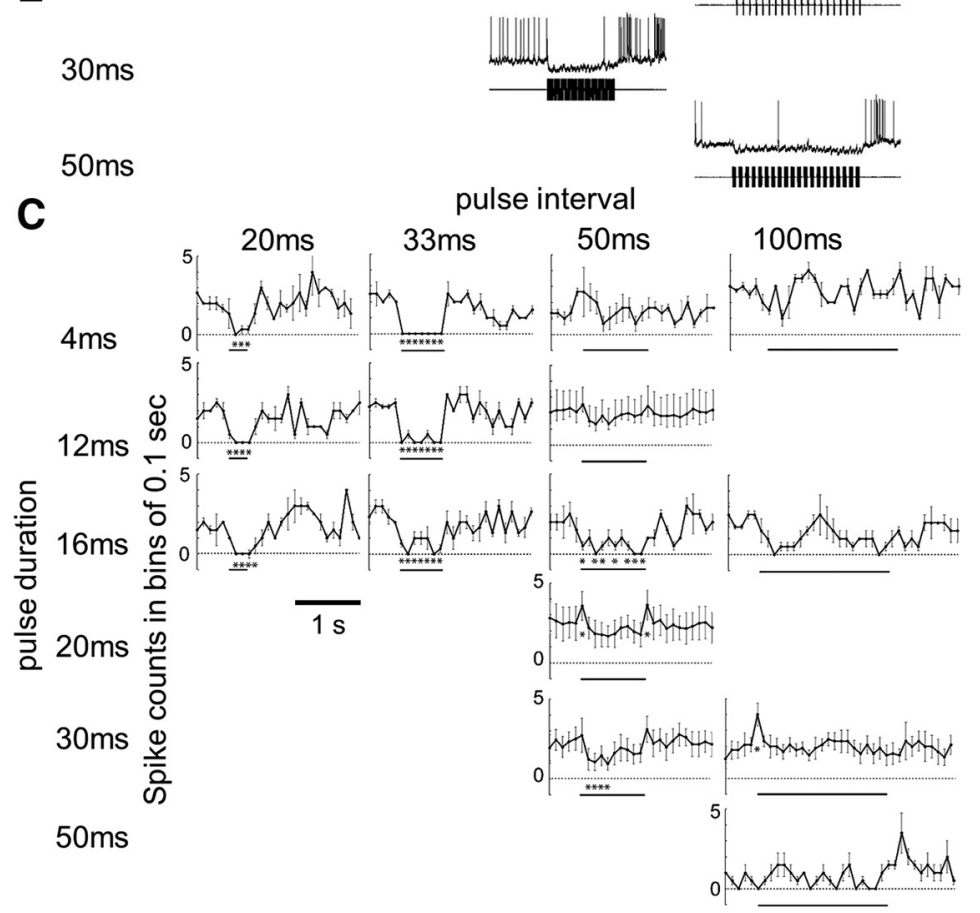

B

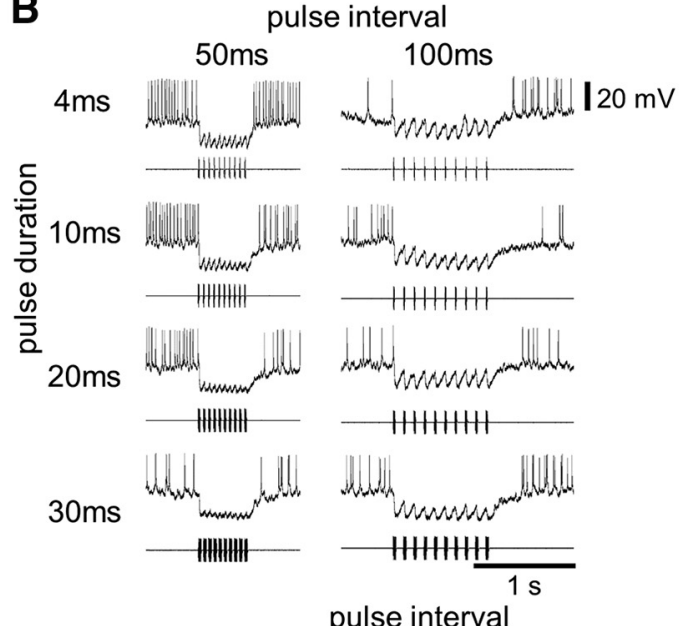

D

$4 \mathrm{~ms}$
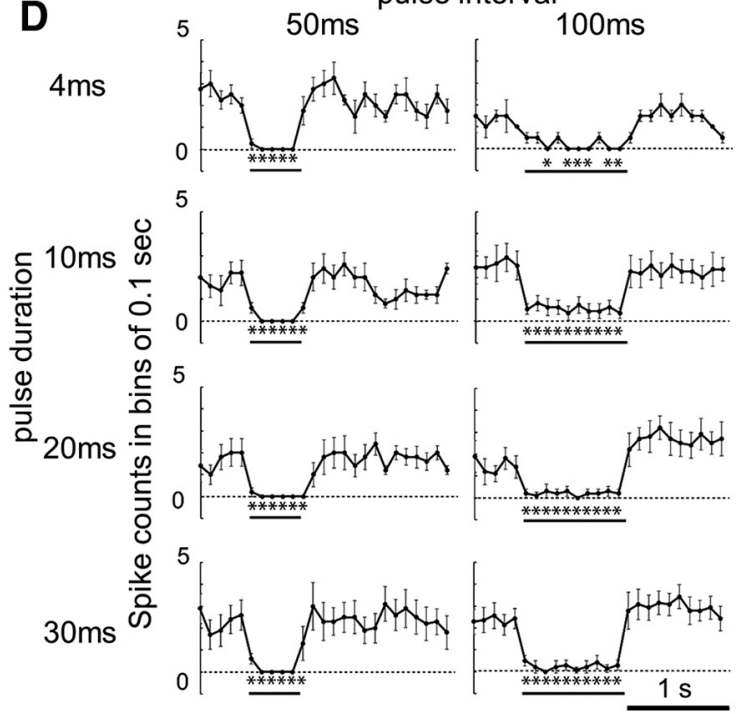

Figure 3. Responses of DL-Int-1 and DL-dSEG-mPPL neurons to trains of vibration pulses. Each of the records in $\boldsymbol{A}$ and $\boldsymbol{B}$ presents the data obtained from one animal. $\boldsymbol{A}, \boldsymbol{B}$, Single responses of two example neurons (preparations HB141121-1AL and HB130226-1Rh) to pulse stimuli applied to the antenna with different temporal patterns. Pulse durations were varied between 4 and $50 \mathrm{~ms}$, and pulse intervals were varied between 20 and $100 \mathrm{~ms}$. The carrier frequency of the pulse vibration was $265 \mathrm{~Hz}$. DL-Int-1 neurons showed tonic inhibitory responses for shorter pulse durations ( $<50 \mathrm{~ms}$ ), while DL-dSEG-mPPL neurons showed this response as well as a response to pulses with a longer pulse duration ( $100 \mathrm{~ms}$ ). $C$, $\boldsymbol{D}$, Instantaneous spike frequencies (spike counts in bins of $0.1 \mathrm{~s}$ ) of DL-Int-1 ( $(\boldsymbol{C}$ and DL-dSEG-mPPL neurons $(\boldsymbol{D} ; N=7)$. The horizontal bars indicate the duration of a train of pulses. Asterisks indicate statistical differences with the spontaneous activity in the $1 \mathrm{~s}$ interval before each record $\left({ }^{*} p<0.01\right)$. DL-Int-1 neurons respond with a tonic inhibitory response to trains of pulses with a pulse interval up to $33 \mathrm{~ms}$, while in DL-dSEG-mPPL neurons we also observed tonic inhibition for longer pulse durations, such as $100 \mathrm{~ms}$. [Note that, owing to experimental conditions, responses to stimulation with shorter pulse durations (20 and $33 \mathrm{~ms}$ ) were not recorded for DL-dSEG-mPPL neurons].

below the spontaneous level, and spikes were typically not observed during stimulation. We only observed intermittent spikes during trains of vibration pulses for pulse trains with long IPIs of $100 \mathrm{~ms}$, which is far above the $14-30 \mathrm{~ms}$ IPI observed during the waggle dance. Statistical analysis revealed that spike rates significantly decreased during stimulation for pulse durations up to $16 \mathrm{~ms}$ and pulse intervals up to $33 \mathrm{~ms}$ (Wilcoxon signed rank test, $n=14$; Fig. $3 C$ ).

Similar to DL-Int-1 neurons, DL-dSEG-mPPL neurons showed tonic inhibitory responses to trains of pulses. These responses were more pronounced for long-pulse intervals of $100 \mathrm{~ms}$ (Fig. 3B). Statistical analysis revealed that spike rates significantly decreased during stimulation for pulse intervals of $\geq 50 \mathrm{~ms}$ (Wilcoxon signed rank test, $n=6$; Fig. $3 D$ ). It is not clear whether this tonic inhibition also occurs for pulse intervals $<50 \mathrm{~ms}$ because data could not be obtained for these stimuli.

In contrast to DL-Int-1 and DL-dSEG-mPPL neurons, excitatory responses were observed in DL-Int-2 neurons (Fig. 4A). Specifically, the temporal response profiles of DL-Int-2 neurons showed remarkable on-phasic excitation up to rates of $>70 \mathrm{~Hz}$, as well as tonic excitatory responses to trains of pulses with durations of $16 \mathrm{~ms}$ and pulse intervals of $33 \mathrm{~ms}$ (Wilcoxon signed rank test, $n=5$; Fig. $4 B$ ). Bilateral DL-dSEG-LP neurons also showed excitatory responses to trains of vibration pulses. In these neurons, each pulse onset elicited a single spike or EPSP, regardless of pulse duration and IPI (Fig. 5).

Response profiles of PAC neurons in response to a single pulse To evaluate the response characteristics of PAC neurons, we analyzed the temporal response patterns induced by single pulses of different durations. We compared the spike counts, spike rates, and latencies of the first spikes of DL-Int-1 (Fig. 6A-E), DL-Int-2 (Fig. $6 F-I$ ), and bilateral DL-dSEG-LP neurons (Fig. 6J, K) evoked by single pulses with different pulse durations. The number of DL-Int-1 spikes elicited by a single vibration pulse gradually increased with the pulse duration up to $20 \mathrm{~ms}$, and was saturated for pulse durations $>20 \mathrm{~ms}(1.1 \pm 0.6$ at a pulse dura- 
A

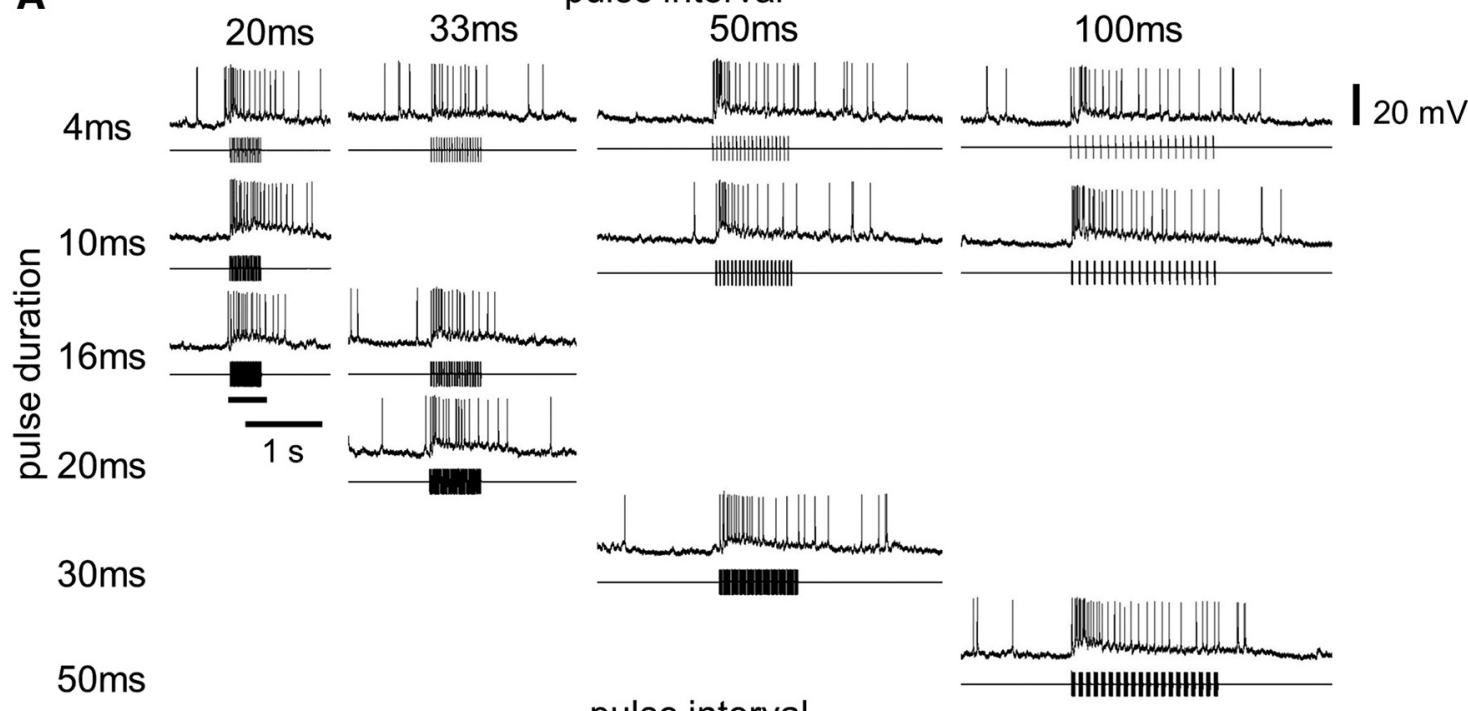

B pulse interval

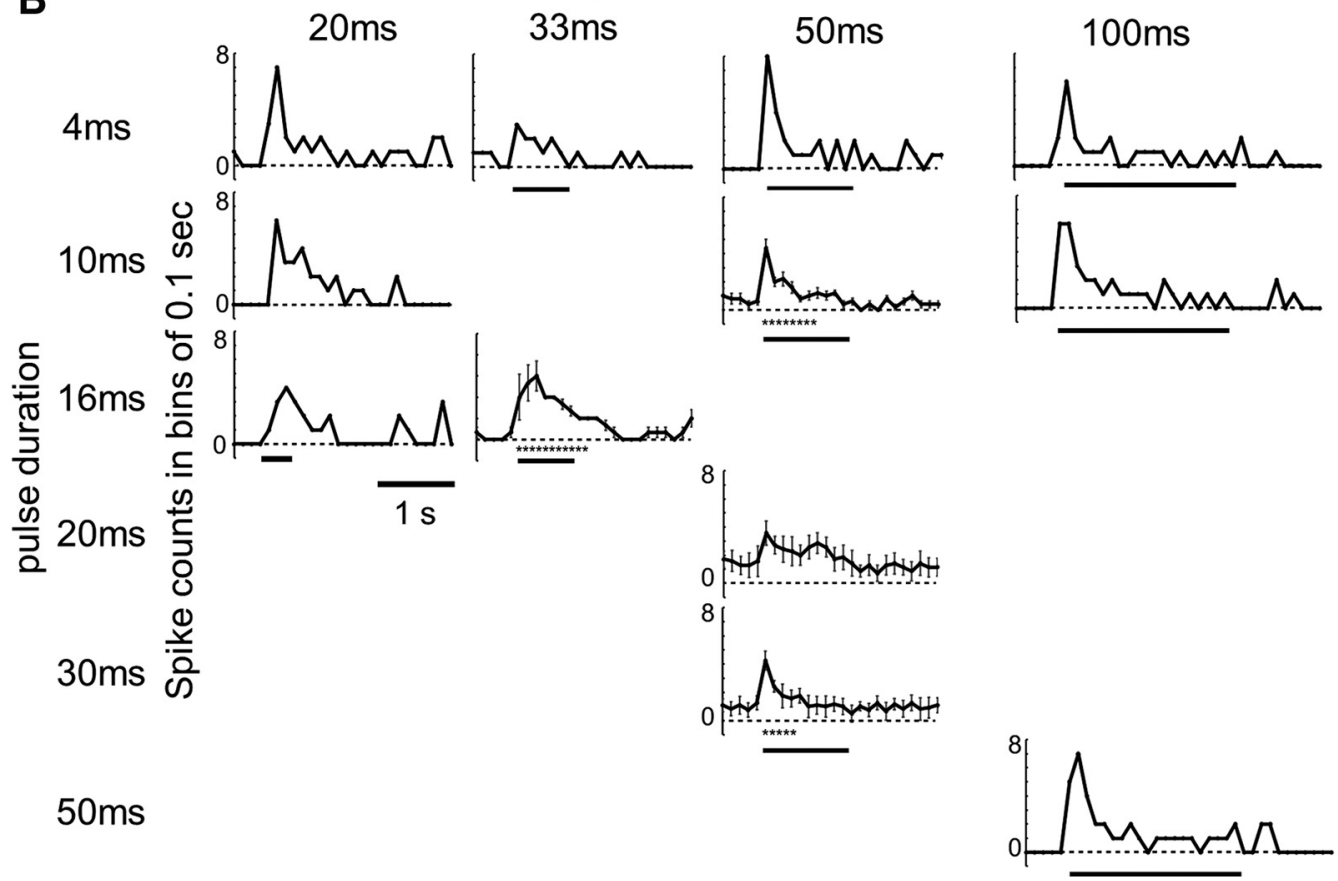

Figure 4. Responses of DL-Int-2 neurons to trains of vibration pulses. A, Single responses of an example neuron to pulse train stimuli applied to the antenna with different temporal patterns (preparation HB140605-2Rh). All records present the data obtained in one animal. DL-Int-2 neurons showed tonic excitatory responses during trains of vibratory pulses with different temporal patterns. $B$, Instantaneous spike frequencies of DL-Int- 2 neurons before, during, and after the train of pulses $(N=6)$. The bin size was $0.1 \mathrm{~s}$. The horizontal bars indicate the duration of a train of pulses. Asterisks indicate statistical differences compared with the spontaneous activity collected just before each record $\left({ }^{*} p<0.01\right)$. DL-Int-2 neurons responded with a tonic excitatory response to a train of pulses with a duration of $16 \mathrm{~ms}$ and interval of $33 \mathrm{~ms}$. [Note that owing to experimental conditions, responses to stimulation with shorter ( $20 \mathrm{~ms}$ ) and longer (100 ms) pulse intervals and with shorter (4 ms) pulse durations were recorded in only one preparation for DL-Int-2.]

tion of $4 \mathrm{~ms}$ and $2.8 \pm 1.0$ at pulse durations of 20,30 , and $50 \mathrm{~ms}$, Fig. $6 B$; Wilcoxon signed rank test, $n=46$ ). However, for values of $>10 \mathrm{~ms}$, the average spike rate for the phasic spikes gradually decreased as the pulse duration increased $(180 \pm 26 \mathrm{~Hz}$ at $10 \mathrm{~ms}$ pulse duration and $134 \pm 53 \mathrm{~Hz}$ at $50 \mathrm{~ms}$ pulse duration; Fig. $6 \mathrm{C}$; Wilcoxon signed rank test, $n=67$ ), indicating the presence of tonic inhibition during the pulse stimulation. The latency of the first DL-Int-1 neuron spike after the onset of a single pulse (Fig. $6 A$ ) was constant, independent of pulse duration, for pulses $<50 \mathrm{~ms}$ (7.5 \pm 1.4 ms; Fig. $6 D$; Wilcoxon signed rank test, $n=34$ ). The latency of the first spike after the offset of a single pulse was also constant, independent of pulse duration, for pulses of $<50 \mathrm{~ms}$
(57 \pm 14 ms; Fig. 6E; Wilcoxon signed rank test, $n=34$ ). For DL-Int-2 neurons, the number of spikes was significantly different for pulse durations of 4 versus $10 \mathrm{~ms}$ and was saturated for values of $>10 \mathrm{~ms}(1.4 \pm 1.0$ at a pulse duration of $4 \mathrm{~ms}$ and $2.8 \pm$ 0.5 for pulse durations of $>10 \mathrm{~ms}$; Fig. $6 G$; Wilcoxon signed rank test, $n=18$ ), while spike rates and spike latency were constant independent of pulse duration (Fig. $6 F, H, I ; 107 \pm 19 \mathrm{~Hz}$ spike frequency and $9.3 \pm 1.7 \mathrm{~ms}$ for the latency of the first spike for DL-Int-2 neuron, Wilcoxon signed rank test, $n=49$ ). The latencies of the EPSPs of bilateral DL-dSEG-LP neurons were also constant, independent of the pulse duration $(5.2 \pm 1.2 \mathrm{~ms}$, Wilcoxon signed rank test; Fig. $6 J, K)$. 


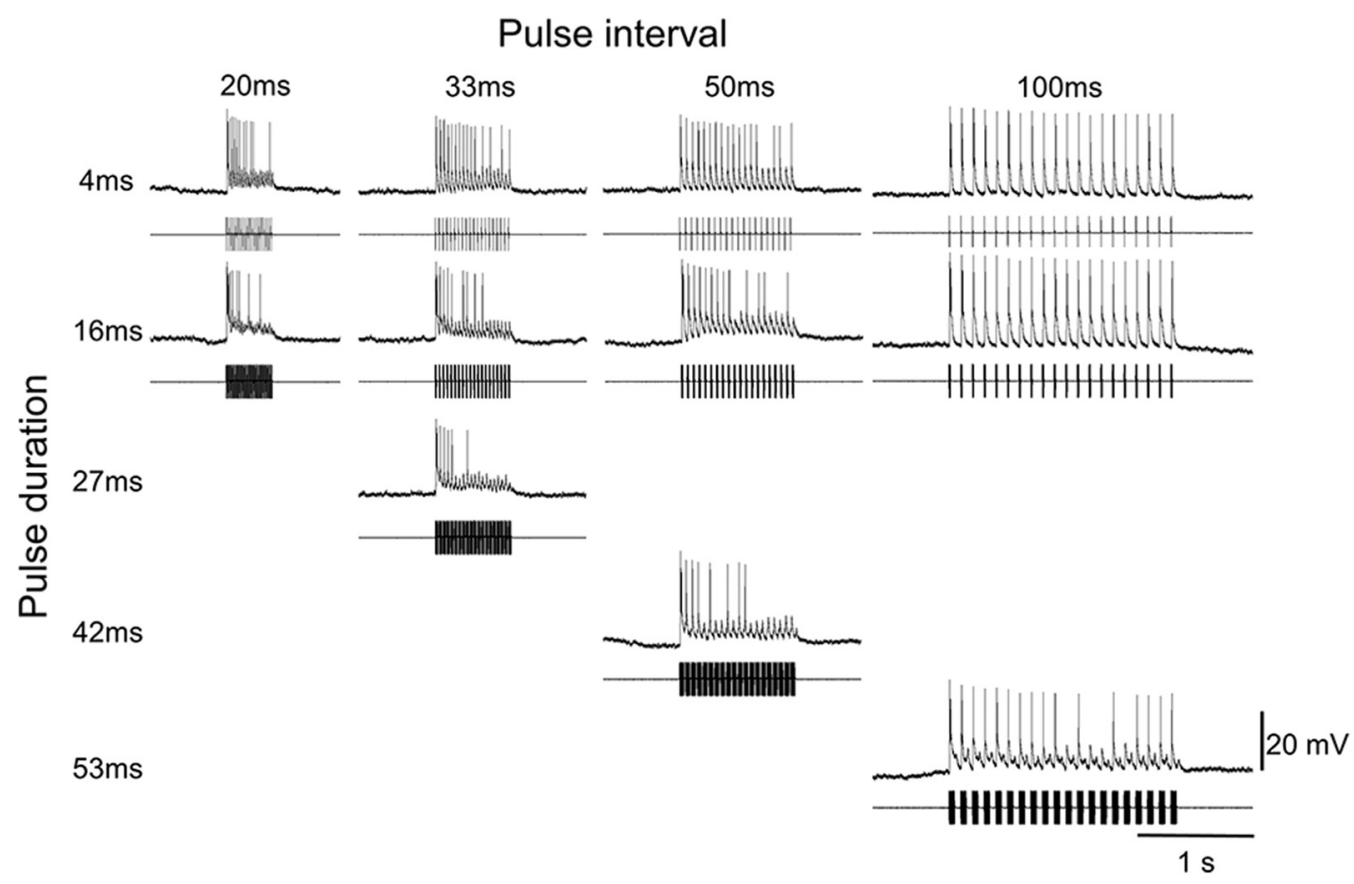

Figure 5. Responses of the bilateral DL-dSEG-LP neuron to trains of vibration pulses. Single responses of an example neuron to pulse train stimuli applied to the antenna with different temporal patterns (preparation HB140522-1AL). All records present the data obtained from the same neuron in one animal. This neuron type does not show spontaneous activity, but in response to pulse stimuli reliably shows EPSPs or spikes. Spikes or EPSPs were phase locked to the vibration pulses, even if the temporal pattern of stimuli changed (Fig. $7 \mathrm{H}, I)$.
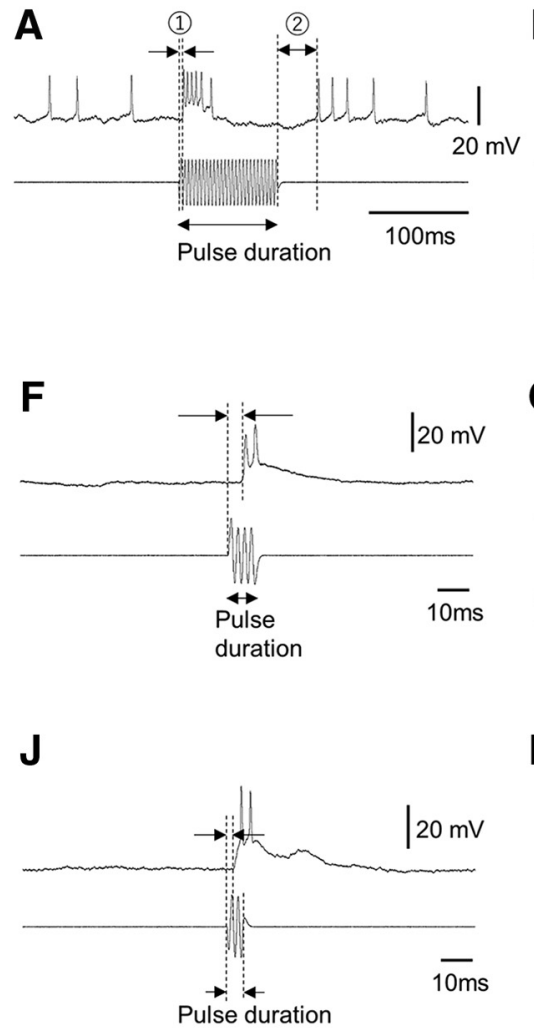

B

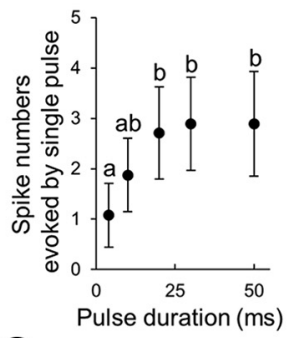

G

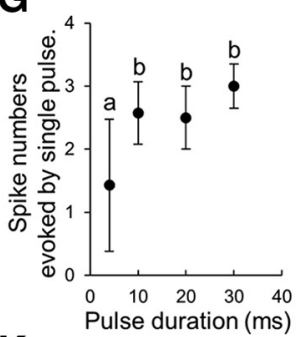

K

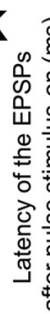

C
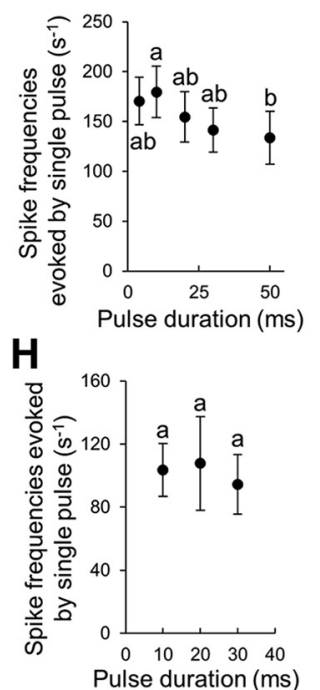

D
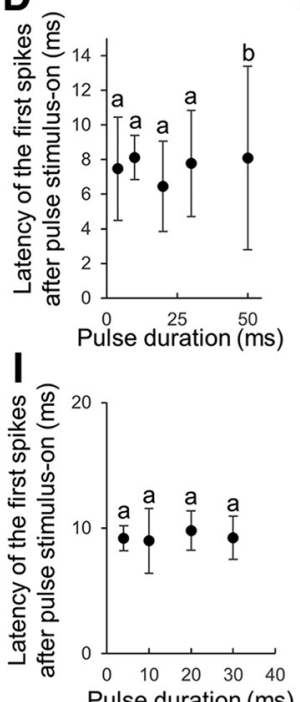

E

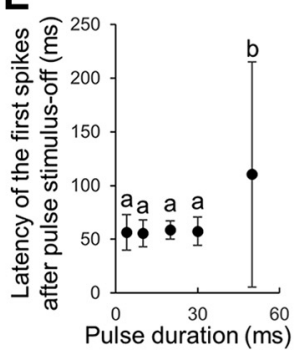

Figure 6. Characteristics of responses evoked by single pulses ( $A-E, D L-I n t-1 ; \boldsymbol{F}-\boldsymbol{I}, \mathrm{DL}-\mathrm{Int}-2 ; \boldsymbol{J}, \boldsymbol{K}$, bilateral DL-dSEG-LP). $\boldsymbol{A}$, Example of the on-off phasic excitation of $\mathrm{DL}-\mathrm{Int}-1 \mathrm{n}$ neuron to a single pulse with a duration $100 \mathrm{~ms}$. Latencies of the spike after onset and offset of the pulse stimulus are indicated by (1) and by (2), respectively. $\boldsymbol{B}, \boldsymbol{C}$, number $(\boldsymbol{B})$ and frequency $(\boldsymbol{C})$ of spikes evoked by a vibration pulse. Within each plot, different letters $(\mathrm{a}, \mathrm{b})$ indicate significant differences between values. $\boldsymbol{D}, \boldsymbol{E}$, Latencies of the spikes after stimulus-on $(\boldsymbol{D})$ and stimulus-off $(\boldsymbol{E})$ are not significantly different for the different pulse durations. $\boldsymbol{F}$, Example of the response of DL-Int-2 neuron to a single pulse with a duration of $10 \mathrm{~ms}$. The latency of the spike after pulse stimulus-on is indicated by the time lag between the two vertical dashed lines. $\mathbf{G}, \boldsymbol{H}$, Spike number $(\boldsymbol{G})$ and spike frequency $(\boldsymbol{H})$ evoked by single pulses with different durations. $\boldsymbol{I}$, the latencies after pulse stimulus-on were not significantly different for different pulse durations. J, Example of the response of a bilateral DL-dSEG-LP neuron to a single pulse with a duration of $10 \mathrm{~ms}$. The latency of the spike after pulse stimulus-on is indicated by the time lag between the two vertical dashed lines. $\boldsymbol{K}$, The EPSP or spike latencies after pulse onset. Latencies were not significantly different for different pulse durations. 

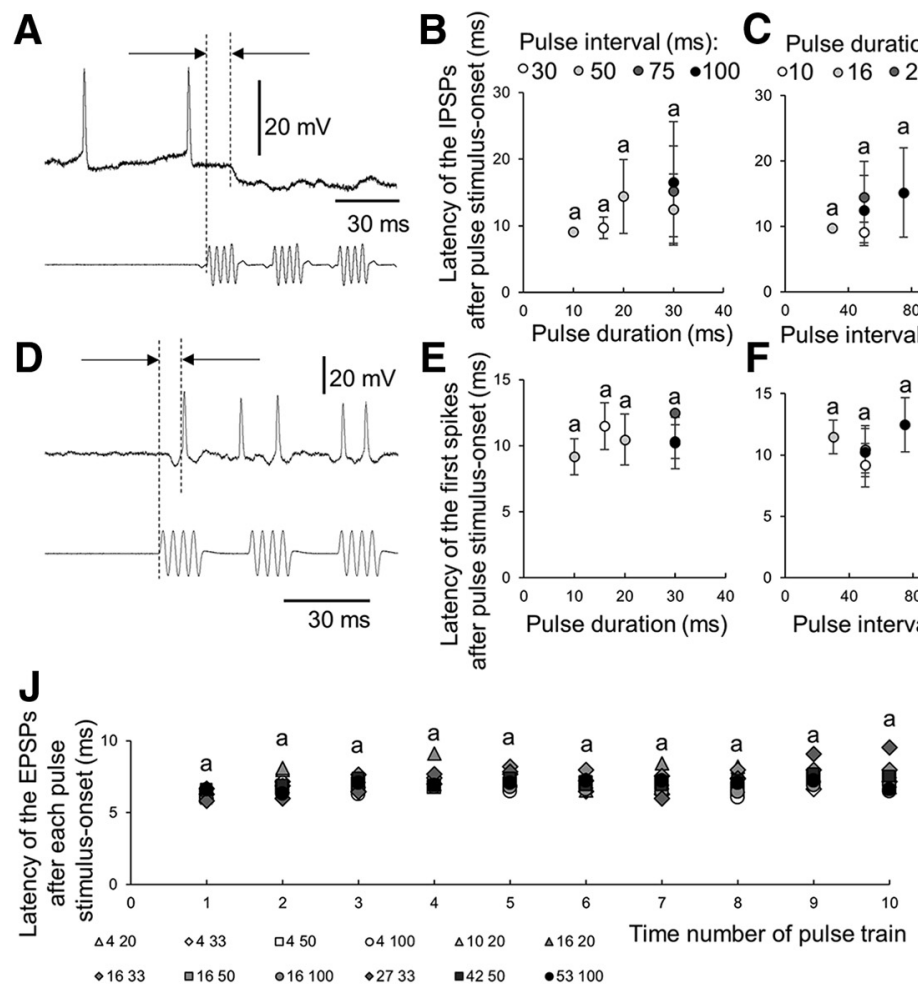

The numbers of each symbol show pulse interval and duration, respectively.
C Pulse duration (ms): $010 \circ 16 \cdot 20 \cdot 30$

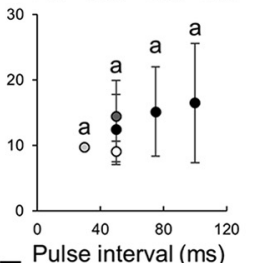

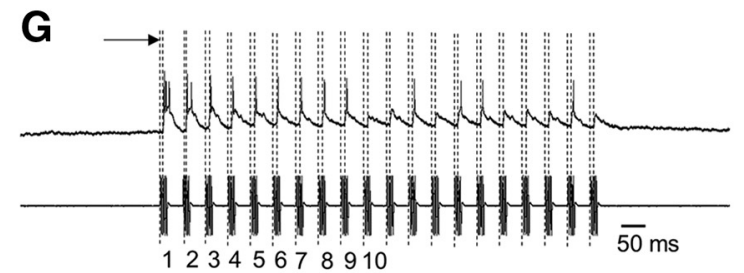

12345678910
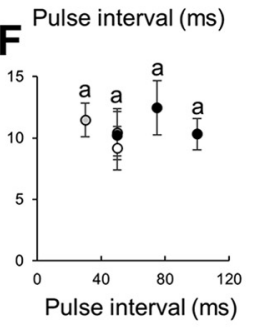
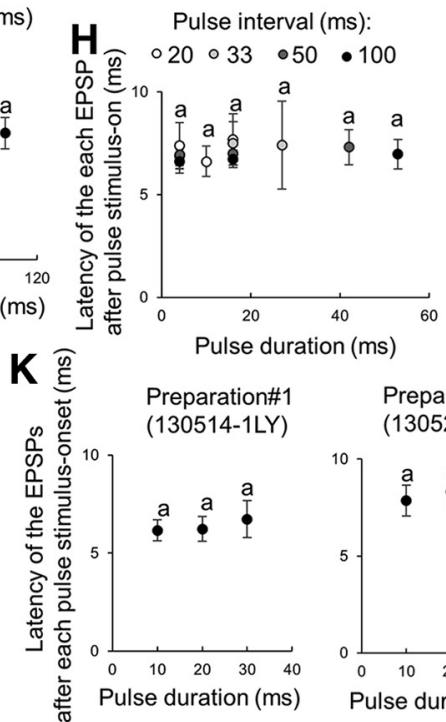
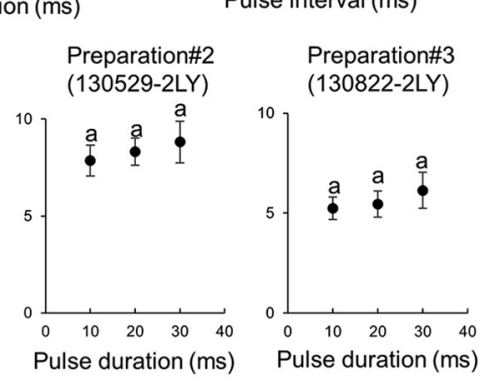

Figure 7. $\quad \boldsymbol{A}-\boldsymbol{K}$, Response characteristics evoked by pulse trains ( $\boldsymbol{A}-\boldsymbol{C}, \mathrm{DL}-\mathrm{Int}-1 ; \mathbf{D}-\boldsymbol{F}, \mathrm{DL}-\mathrm{Int}-2 ; \boldsymbol{G}-\boldsymbol{K}$, bilateral DL-dSEG-LP neurons). $\boldsymbol{A}$, Example of the first IPSP of the DL-Int-1 neuron evoked by a train of $15 \mathrm{~ms}$ pulses. The latency of the IPSP is indicated by the time lag between the two vertical dashed lines. $\boldsymbol{B}, \boldsymbol{C}$, The mean latencies of the IPSPs over recordings from different preparations were not significantly different for different pulse durations $(\boldsymbol{B})$ or pulse intervals $(\boldsymbol{C})$. $\boldsymbol{D}$, Example of the first spike of a DL-Int-2 neuron induced by a train of $15 \mathrm{~ms}$ pulses. The latency of the first spike is indicated by the time lag between the two vertical dashed lines. $\boldsymbol{E}, \boldsymbol{F}$, latencies were not significantly different for different pulse durations $(\boldsymbol{E})$ or pulse intervals $(\boldsymbol{F})$. $\boldsymbol{G}$, Example of the response of a bilateral DL-dSEG-LP neuron to a train of $20 \mathrm{~ms}$ pulses. The latencies of the EPSPs after the pulse stimulus-on is indicated by the pairs of vertical dashed lines. $\boldsymbol{H}, \boldsymbol{I}$, The latencies of the EPSPs after pulse stimulus-on were not significantly different for different pulse durations $(\boldsymbol{H})$ or intervals $(\boldsymbol{I})$. $\boldsymbol{J}$, latencies of the EPSPs after each pulse stimulus-on in a train of pulses (no significant differences). $\boldsymbol{K}$, EPSP latencies of three different neurons for different pulse durations (no significant differences).

Response profiles of PAC neurons in response to pulse trains For stimulation with trains of vibration pulses, the latencies of the first IPSPs of DL-Int-1 neurons (Fig. $7 \mathrm{~A}$ ) were constant $(13.3 \pm$ $6.3 \mathrm{~ms}$ ), independent of either pulse duration (Fig. $7 B$; Wilcoxon signed rank test, $n=35$ ) or pulse interval (Fig. 7C; Wilcoxon signed rank test, $n=35$ ). The first spike latencies of DL-Int-2 neurons (Fig. $7 D$ ) were also constant, independent of pulse duration (10.4 $\pm 2.0 \mathrm{~ms}$; Fig. $7 E$; Wilcoxon signed rank test, $n=35$ ) or pulse interval (Fig. 7F; Wilcoxon signed rank test, $n=35$ ). Bilateral DL-dSEG-LP neurons showed no spontaneous activity, but trains of vibration pulses applied to the antenna reliably elicited spikes or EPSPs during the pulsed vibration (Fig. $7 G$ ). The spikes or EPSPs corresponded in a one-to-one fashion to the vibration pulses and followed the pulses even if the temporal pattern of the stimulus changed. This indicates that bilateral DLdSEG-LP neurons generate phase-locked spikes or EPSPs in response to trains of vibration pulses. EPSP latencies during a train of pulses were constant (20 pulse trains for each, $n=5$ ), independent of pulse duration (Fig. 7H; Wilcoxon signed rank test, $n=20$ ), pulse interval (Fig. 7I; Wilcoxon signed rank test, $n=$ 20 ), or the number of pulses in the train (Fig. 7J; Wilcoxon signed rank test, $n=10)$. However, these latencies showed slight variations depending on individual preparations $(6.4 \pm 0.8 \mathrm{~ms}$ for preparation 130514-1LY; $8.3 \pm 1.0 \mathrm{~ms}$ for preparation $130529-$ $2 \mathrm{LY} ; 5.6 \pm 0.8 \mathrm{~ms}$ for preparation $130822-2 \mathrm{LY}$; Fig. $7 \mathrm{~K} ; p<0.01$, Wilcoxon-signed rank test; 10 pulses for each individual).

\section{Model of inhibitory network in the PAC}

The projection patterns of DL-Int-1 and DL-Int-2 (Fig. 8A) along with their immunocytochemistry (Fig. 2) and response properties, including their relative onset latencies to single-pulse stimulations (Fig. $7 A, D, F, I$ ), suggest a network with inhibitory connections from DL-Int-1 to DL-Int-2. (Fig. $8 B, C$ ). The observed response properties of DL-Int-2, with tonic excitation decreasing for increasing pulse intervals, could be a result of disinhibition due to the decreasing tonic inhibition of DL-Int-1 (Fig. 8D). To test the plausibility of such a network in the PAC, we implemented a model of DL-Int- 1 and DL-Int-2 neurons in a circuitry with corresponding excitatory and inhibitory connections (Fig. 8C) and investigated its responses to continuous and pulse train sinusoidal stimuli at waggle dance frequency $(265 \mathrm{~Hz})$.

When continuous vibration stimuli were used, model DLInt-1 neurons in the network showed phasic excitation in response to vibration onset, followed by spikeless tonic inhibition (data not shown), in accordance with known properties of DLInt-1 neurons (Ai et al., 2009). When the network was stimulated with vibration pulses with pulse intervals $\sim 30 \mathrm{~ms}$, similar to waggle dance vibration pulses, model DL-Int-1 neurons exhibited no spikes during stimulation and weak rebound spikes after stimulation offset (Fig. $8 E$ ). When vibration pulses were separated by longer pulse intervals $\sim 100 \mathrm{~ms}$, intermediate spikes occurred during stimulation pulses (Fig. 8E), similar to what was observed experimentally (Fig. 8D). Model DL-Int-2 neurons showed on- 

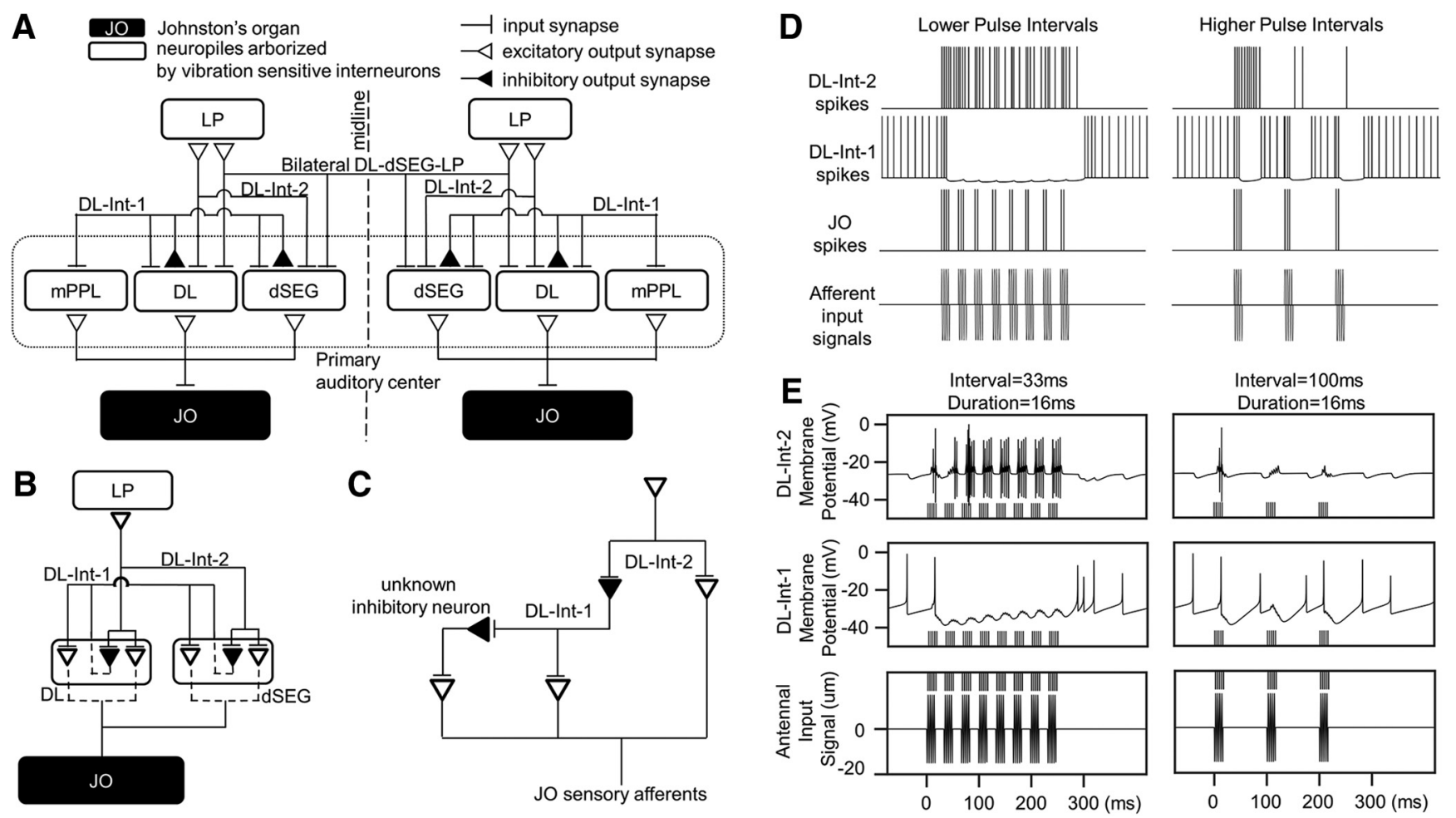

Figure 8. PAC network model based on the arborization and response patterns of interneurons. A, Summary of projection patterns of neurons arborizing in the PAC. Since DL-Int- 1 neurons project to the DL-dSEG, where DL-Int-2 neurons arborize, DL-Int-1 neurons could have a synapse onto DL-Int-2 neurons. $\boldsymbol{B}$, Subset of neural projections in $\boldsymbol{A}$ with putative synaptic connections (dotted lines). DL-Int-1 and DL-Int-2 neurons are assumed to have direct excitatory input from J0 sensory afferents in the DL and dSEG. Since DL-Int- 1 neurons are GABAergic, DL-Int-2 neurons are assumed to have an inhibitory synapse from DL-Int-1 in the DL and dSEG. $\boldsymbol{C}$, Neurons and synapses in $\boldsymbol{B}$ represented as a network model. Synapses shown in $\boldsymbol{B}$ indicating the same connectivity in the $D L$ and dSEG are represented by single synapses. An unknown inhibitory neuron is added between J0 and DL-Int- 1 to account for its inhibitory response. $D$, Summary of the experimental physiology of JO (bottom), DL-Int-1 (middle), and DL-Int-2 (top) neurons shown using schematic membrane traces for stimuli with shorter ( $\sim 30 \mathrm{~ms}$, left column) and longer ( $~ 100 \mathrm{~ms}$, right column) pulse interval values. Note that the shorter intervals correspond to the vibration elicited during the honeybee waggle dance. J0 sensory neurons tend to spike at a fixed phase of the input sinusoidal stimulus, showing spike frequency adaptation for later pulses. DL-Int-1 neurons show inhibition that is stronger for stimuli with shorter pulse intervals than those for stimuli with longer pulse intervals, and intermittent spikes occur during a train of pulses for long pulse intervals. DL-Int-2 neurons show on-phasic and tonic excitation, with the latter being weaker for stimuli with longer pulse intervals, suggesting that it arises from disinhibition due to the tonic inhibition of DL-Int-1. $\boldsymbol{E}$, Simulation results of the network model in C for the same stimuli as in $\boldsymbol{D}$. J0 sensory neurons were assumed to spike regularly at a fixed phase of the sinusoidal stimulus applied to the antenna (bottom row). These spikes are indicated by vertical lines at the top in the bottom row and at the bottom in the middle and top rows. DL-Int-2 shows subthreshold EPSPs evoked by disinhibition through DL-Int-1. The network model could qualitatively reproduce the different spiking profiles for the two stimulus conditions.

phasic excitation and tonic spiking responses during pulse train stimulation with waggle dance-like short pulse intervals (Fig. 8E). For pulse trains with longer pulse intervals, the tonic component was reduced, and, while subthreshold EPSPs were present during stimulation, spikes occurred only sporadically (Fig. $8 E$ ). Thus, like for DL-Int-1, the response behavior of model DL-Int-2 neurons was similar to the experimentally observed responses. In summary, the activity of the model neurons qualitatively reproduced the experimental findings, indicating that the proposed network is compatible with the data.

\section{Discussion}

Extending previous work (Ai et al., 2009; Ai and Itoh, 2012), we investigated three main types of interneurons, DL-Int-1, DLInt-2, and bilateral DL-dSEG-LP, using vibration pulses similar to those produced by honeybees during their waggle dance. DLInt-1 neurons showed on-off phasic excitation and tonic inhibition, while DL-Int-2 and bilateral DL-dSEG-LP neurons showed on-phasic excitation. While the temporal responses differed qualitatively between these neuron types, their quantitative properties, including spike count, rate, and latencies, were largely independent of pulse parameters, suggesting that these auditory interneurons have a stereotyped response pattern. In particular, bilateral DL-
dSEG-LP neurons retained a precise response pattern, even throughout stimulation with trains of pulses. This indicates that the PAC might encode the temporal patterns of the sound produced by the waggle dance, along with other information that requires precise timing, such as the body angle of the follower relative to the direction of the waggle dance (see below).

That DL-Int-1 neurons exhibited a specific temporal response with a spike count that increased with pulse duration for short pulses but produced a constant number of evoked spikes for pulses $>20$ ms (Fig. $6 B$ ) suggests that these neurons receive not only excitatory input from JO afferents but also slow inhibitory input from a yet unknown inhibitory interneuron (Fig. 8C). This is also consistent with the disappearance of the on-off phasic excitation for pulse trains with short pulse intervals (Fig. $3 A, C$ ). The latencies of the first spikes produced by DL-Int-2 neurons (Fig. 6I), which were also constant and longer than those of DLInt-1 neurons (Fig. $6 D$ ), suggest that DL-Int-2 neurons might receive input from DL-Int-1 neurons.

\section{Possible neural mechanisms of the encoding of waggle dance information}

Based on the current and previous experimental findings, we can speculate about the possible neural mechanisms underlying the 
response behavior of the identified vibration-sensitive interneurons to waggle dance-like trains of vibration pulses.

\section{Distance information}

The duration of the waggle phase increases with the distance to the food source (von Frisch, 1967). During the waggle phase, the dancer produces vibration pulses during the moments at which her tail end passes in front of the follower. There are two possible parameters for encoding the distance: the duration of the train of vibration pulses and the number of pulses.

Based on the responses of DL-Int-1 neurons to continuous vibration, we previously proposed (Ai et al., 2009) that these neurons could encode the duration of vibration during the waggle phase. The present results demonstrate that the DL-Int-1 neuron also shows tonic inhibition during trains of vibration pulses and that it is an inhibitory interneuron, while the response pattern of the DL-Int-2 neuron is characterized by tonic excitation to a train of vibration pulses. Both neurons show precise latencies after the onset of a vibration pulse train (Fig. 7), but the response to stimulus offset is more precise in DL-Int-1 neurons than in DL-Int-2 neurons. This indicates that DL-Int-1 neurons are more accurate in encoding the waggle phase duration and that DL-Int-2 neurons might be postsynaptic to DL-Int-1 neurons. A previous spatial proximity analysis of dendrites indicated that DL-Int-2 neurons might receive direct input from JO afferents (Ai, 2010). Together, this may suggest that DL-Int-2 neurons receive disinhibitory input through DL-Int-1 neurons. As our model simulations (Fig. 8) indicate, the responses of these neurons are consistent with the presence of inhibition from DL-Int-1 neuron to DL-Int-2 neuron; however, further studies are needed to experimentally assess the synaptic contacts and transmission properties of PAC neurons. If honeybees use the duration of the waggle phase to describe the distance to the food source, then DL-Int- 1 and DL-Int-2 neurons are potential candidates for processing this information.

Bilateral DL-dSEG-LP neurons reliably produced spikes or EPSPs in response to each pulse in a train-of-pulses stimulus and followed the vibration pulses even if the temporal pattern of the stimuli changed. The short onset latency suggests that this type of neuron receives direct inputs from JO afferents of both antennae. If honeybees use the number of pulses to encode distance, then the bilateral DL-dSEG-LP neuron is a potential candidate for processing this information. However, there is also a possible role for the bilateral DL-dSEG-LP neuron in encoding direction (see below).

\section{Direction information}

The waggle dancer communicates the direction of a profitable flower by the angle of the waggle run relative to the vertical, corresponding to the angle between the direction of the food source and the sun (von Frisch, 1967). The receivers of the information, the follower bees, are located at various positions around the line of wagging of the dancer and therefore must determine the difference between their body angle and that of the dancer during the waggle phase to decode the direction. Exactly how the follower bees achieve this computation is unclear. Michelsen (2003) hypothesized that the follower could perceive its orientation relative to the wagging run by assessing differences in the temporal patterns of airflow, specifically in terms of the difference in the intervals of the vibration pulses between both antennae. Such processing would require highly precise encoding and comparison of very short time lags. Since the bilateral DL-dSEG-LP neuron shows relatively precise spike timing in response to vibration pulses, this neuron is a candidate for encoding information about the direction of the food source. To test this possibility, it is necessary to clarify whether the bilateral DL-dSEG-LP neuron can encode differences between the timing of the airflow at the two antennae.

\section{Significance of an inhibitory network in the DL}

DL-Int-1 IPSPs tend to occur with similar latencies as DL-Int-2 spikes (Fig. 7A-F). It is possible that a synaptic contact connects DL-Int-1 neurons to DL-Int-2 neurons and that the tonic excitation of DL-Int-2 neurons is caused by disinhibition from DL-Int-1 neurons. Interestingly, an inhibitory network has been found in the primary olfactory center [i.e., the antennal lobe $(\mathrm{AL})$ ] of the tobacco horn moth (Christensen et al., 1998), where a GABAergic inhibitory local interneuron induces phase-locked bursts of projection neurons encoding intermittent odor stimuli. An analogous inhibitory network might exist in the honeybee, consisting of DL-Int-1 and DL-Int-2 neurons, for encoding waggle dance vibration pulses.

The network model (Fig. $8 B, C$ ) qualitatively replicated the experimentally observed differences in the response behavior of DL-Int- 1 and DL-Int-2 neurons (Fig. $8 D, E$ ). Thus, although further studies are needed to model the responses of these neurons in more quantitative detail, these results support the existence of an inhibitory network in the PAC.

\section{Comparison of vibration-processing interneurons between the honeybee and other invertebrates}

Invertebrate sensory processing typically consists of a rapid transition from coarse representations into perceptual and behavioral features over relatively few processing stages (Hildebrandt, 2014). It is very likely that a similar strategy is used for processing auditory signals in the honeybee. Our results indicate that neurons encoding different types of features arborize in the same region, the DL. Bilateral DL-dSEG-LP neurons closely follow stimuli with phaselocked spike responses, while the responses of DL-Int-1 and DLInt-2 neurons are tuned to the behaviorally relevant features of waggle dance communication.

The interplay between excitation, delayed inhibition, and the resulting rebound depolarization have been shown in several species to be important in neural circuits that extract temporal features of sensory signals (Pollack, 2001; Large and Crawford, 2002; Alluri et al., 2016; Schöneich et al., 2015). In the honeybee, the DL-Int-1 neuron has a response consisting of on-phasic excitation, tonic inhibition, and rebound depolarization, which could result from the superimposition of an excitation and a delayed inhibition. These characteristics strongly indicate that the DLInt-1 neuron is part of a network that extracts distance information that is temporally encoded in waggle dance communication signals.

A number of vibration-processing interneurons and neural pathways have been comprehensively identified in Drosophila, particularly in the PAC and the antennal mechanosensory and motor center (Kamikouchi et al., 2006; Lai et al., 2012; Vaughan et al., 2014; Matsuo et al., 2016). Some of these pathways are related to courtship song detection (Vaughan et al., 2014).

The male Drosophila produces an airborne vibration with a species-specific pulse song for attracting conspecific females (Ewing and Bennet-Clark, 1968; Cowling and Burnet, 1981). The honeybee uses pulses of airborne vibration for waggle dance communication. This analogy between the auditory behaviors of these two species suggests the presence of common characteristics in the central processing of vibration pulses. Comparison of the neurons involved in processing vibration signals in the two insects reveals a 
number of common characteristics, such as inhibitory networks in the PAC, a secondary auditory center located in the lateral protocerebrum, which is also the secondary olfactory center, and bilateral interneurons connecting the PACs of both hemispheres.

However, further research is necessary to conclusively determine the role of interneurons in courtship song detection in the fly as well as the roles of DL-Int-1, DL-Int-2, and bilateral DLdSEG-LP neurons in the encoding of vector information in the honeybee. Specifically, we hope to clarify the precise neural circuitry underlying the encoding of waggle dance vector information in the honeybee brain.

\section{References}

Ai H (2010) Vibration-processing interneurons in the honeybee brain. Front Syst Neurosci 3:19. CrossRef Medline

Ai H, Hagio H (2013) Morphological analysis of the primary center receiving spatial information transferred by the waggle dance of honeybees. J Comp Neurol 521:2570-2584. CrossRef Medline

Ai H, Itoh T (2012) The Auditory System of the Honeybee. In Honeybee neurobiology and behaviors, Ed 2 (Eisenhardt D, Galizia CG, Giurfa M, eds), pp 269-284. Berlin/Heidelberg, Germany: Springer.

Ai H, Nishino H, Itoh T (2007) Topographic organization of sensory afferents of Johnston's organ in the honeybee brain. J Comp Neurol 502:10301046. CrossRef Medline

Ai H, Rybak J, Menzel R, Itoh T (2009) Response characteristics of vibrationsensitive interneurons related to Johnston's organ in the honeybee, Apis mellifera. J Comp Neurol 515:145-160. CrossRef Medline

Alluri RK, Rose GJ, Hanson JL, Leary CJ, Vasquez-Opazo GA, Graham JA, Wilkerson J (2016) Phasic, suprathreshold excitation and sustained inhibition underlie neuronal selectivity for short-duration sounds. Proc Natl Acad Sci U S A 113:E1927-E1935. CrossRef Medline

Brette R, Gerstner W (2005) Adaptive exponential integrate-and-fire model as an effective description of neuronal activity. J Neurophysiol 94:36373642. CrossRef Medline

Brockmann A, Robinson GE (2007) Central projections of sensory systems involved in honey bee dance language communication. Brain Behav Evol 70:125-136. CrossRef Medline

Carnevale NT, and Hines ML (2006) The NEURON book. Cambridge, UK: Cambridge UP.

Christensen TA, Waldrop BR, Hildebrand JG (1998) Multitasking in the olfactory system: context-dependent responses to odors reveal dual GABA-regulated coding mechanisms in single olfactory projection neurons. J Neurosci 18:5999-6008. Medline

Cowling DE, Burnet B (1981) Courtship songs and genetic control of their acoustic characteristics in sibling species of the Drosophila melanogaster subgroup. Anim Behav 29:924-935. CrossRef

Dreller C, Kirchner WH (1993) Hearing in honeybees: localization of the auditory sense organ. J Comp Physiol A 173:275-279. CrossRef

Ewing AW, Bennet-Clark HC (1968) The courtship songs of Drosophila. Behaviour 31:288-301. CrossRef
Hildebrandt KJ (2014) Neural maps in insect versus vertebrate auditory systems. Curr Opin Neurobiol 24:82-87. CrossRef Medline

Hrncir M, Maia-Silva C, Mc Cabe SI, Farina WM (2011) The recruiter's excitement-features of thoracic vibrations during the honey bee's waggle dance related to food source profitability. J Exp Biol 214:4055-4064. CrossRef Medline

Judd TM (1995) The waggle dance of the honey bee: which bees following a dancer successfully acquire the information? J Insect Behav 8:343-354.

Kamikouchi A, Shimada T, Ito K (2006) Comprehensive classification of the auditory sensory projections in the brain of the fruit fly Drosophila melanogaster. J Comp Neurol 499:317-356. CrossRef Medline

Kirchner WH, Dreller C, Towne WF (1991) Hearing in honeybees: operant conditioning and spontaneous reactions to airborne sound. J Comp Physiol A 168:85-89. CrossRef

Lai JS, Lo SJ, Dickson BJ, Chiang AS (2012) Auditory circuits in the Drosophila brain. Proc Natl Acad Sci U S A 109:2607-2612. CrossRef Medline

Large EW, Crawford JD (2002) Auditory temporal computation: interval selectivity based on post-inhibitory rebound. J Comput Neurosci 13 2:125-142.

Matsuo E, Seki H, Asai T, Morimoto T, Miyakawa H, Ito K, Kamikouchi A (2016) Organization of projection neurons and local neurons of the primary auditory center in the fruit fly Drosophila melanogaster. J Comp Neurol 524:1099-1164. CrossRef Medline

Michelsen A (2003) Karl von Frisch lecture. Signals and flexibility in the dance communication of honeybees. J Comp Physiol A Neuroethol Sens Neural Behav Physiol 189:165-174. CrossRef Medline

Milde JJ (1988) Visual responses of interneurones in the posterior median protocerebrum and the central complex of the honeybee Apis mellifera. J Insect Physiol 34:427-436. CrossRef

Mobbs PG (1982) The brain of the honeybee Apis mellifera. I. The connections and spatial organization of the mushroom bodies. Philos Trans $\mathrm{R}$ Soc Lond B Biol Sci 298:309-354. CrossRef

Pollack GS (2001) Analysis of temporal patterns of communication signals. Curr Opin Neurobiol 11:734-738. CrossRef Medline

Schöneich S, Kostarakos K, Hedwig B (2015) An auditory feature detection circuit for sound pattern recognition. Sci Adv 1:e1500325. CrossRef Medline

Stimberg M, Goodman DF, Benichoux V, Brette R (2014) Equationoriented specification of neural models for simulations. Front Neuroinform 8:6. CrossRef Medline

Towne WF, Kirchner WH (1989) Hearing in honey bees: detection of airparticle oscillations. Science 244:686-688. CrossRef Medline

Tsujiuchi S, Sivan-Loukianova E, Eberl DF, Kitagawa Y, Kadowaki T (2007) Dynamic range compression in the honey bee auditory system toward waggle dance sounds. PLoS One 2:e234. CrossRef Medline

Vaughan AG, Zhou C, Manoli DS, Baker BS (2014) Neural pathways for the detection and discrimination of conspecific song in. D. melanogaster. Curr Biol 24:1039-1049. CrossRef Medline

von Frisch K (1967) The tail-wagging dance as a means of communication when food sources are distant. In: The dance language and orientation of bees (von Frisch K, ed) pp 57-235. Cambridge, MA: Harvard UP. 